\title{
A revised description of Gyrodactylus cichlidarum Paperna, 1968 (Gyrodactylidae) from the Nile tilapia, Oreochromis niloticus niloticus (Cichlidae), and its synonymy with $G$. niloticus Cone, Arthur et Bondad-Reantaso, 1995
}

\author{
Adriana García-Vásquez ${ }^{1}$, Haakon Hansen ${ }^{2,3}$ and Andrew P. Shinn ${ }^{1}$ \\ ${ }^{1}$ Institute of Aquaculture, University of Stirling, Stirling FK9 4LA, UK; \\ ${ }^{2}$ Natural History Museum, Department for Zoology, University of Oslo, P.O. Box 1172, Blindern, NO-0318 Oslo, Norway; \\ ${ }^{3}$ National Veterinary Institute, Section for Parasitology, P.O. Box 8156 Dep., NO-0033 Oslo, Norway
}

Key words: Gyrodactylus cichlidarum, parasite, tilapia, taxonomy, cichlid

\begin{abstract}
A recent infestation of Gyrodactylus cichlidarum Paperna, 1968 on yolk sac fry of Nile tilapia, Oreochromis niloticus niloticus (L.), in an isolated aquarium system in the UK resulted in high mortalities and provided an opportunity to study this species in greater detail. A tentative identification was made using the measurements and drawings of the ventral bar and hamuli provided in the original description; however, details on the morphology of the marginal hooks were lacking. A comparison of the gyrodactylid material collected from $O$. n. niloticus with the holotype of G. cichlidarum, the only known available specimen, from Mango tilapia, Sarotherodon galilaeus galilaeus (L.), confirmed its identity. Proteolytic digestion and image analysis of the opisthaptoral hard parts were used to obtain tissue-free, accurate measurements as part of a complete revised description of $G$. cichlidarum. Further, a comparison of G. cichlidarum from both hosts with the holotype and several paratypes of Gyrodactylus niloticus Cone, Arthur et Bondad-Reantaso, 1995 cited as parasitizing captive stocks of Nile tilapia in the Philippines revealed the two species to be synonymous. An 803 bp fragment of the ribosomal internal transcribed spacers 1 and 2 and the 5.8S was obtained and is provided with the revised description. This is the first DNA sequence from a Gyrodactylus species originating from the African continent. The sequence is very divergent from other species in the genus and only the $5.8 \mathrm{~S}$ sequence places it unambiguously in the genus Gyrodactylus. In addition to G. cichlidarum, two specimens of another morphological similar species of Gyrodactylus were also found on the UK held stock of $O$. n. niloticus. These latter specimens, Gyrodactylus sp., differed from G. cichlidarum in having a longer hamulus point with a smaller hamulus aperture and possessing marginal hook sickles that had a shorter shaft with a longer point giving the sickles a more rounded, closed appearance.
\end{abstract}

The global tilapine (Cichlidae) production currently is ca. 1.5 million tons per annum, an amount second only to farmed carp species (Fitzsimmons 2000, Cutland 2003). The largest producers in 2001 were China (671,666 tons), Egypt (152,515 tons), Thailand (98,250 tons) and Mexico (69,181 tons) (FAO Fishstat plus 2004). Increased productivity and market availability has contributed to its popularity, and in the United States, tilapia is now the ninth most commonly consumed fish species (Fitzsimmons 2000, Cutland 2003). Under intensive culture conditions, however, a wide range of disease organisms can establish quickly and result in large-scale mortalities. Of the possible parasitic disease-causing organisms, ectoparasitic Gyrodactylus Nordmann, 1832 (Monogenea) species are of potential significance to tilapine culture. Clinical outbreaks of gyrodactylosis have been recorded in pond-reared tilapia in Uganda (Fryer and Iles 1972), Colombia (F. Castillo and R. Ramírez, private tilapia producer, pers. comm.), Kenya (Roberts and Sommerville 1980), Thailand (W. Turner, private tilapia producer, pers. comm.), Mexico (V. Vidal-Martínez, CINVESTAV, pers. comm.), Kuwait (M. Al-Marzouk, Kuwait Institute for Scientific Research, pers. comm.), Israel (N. Froyman, private tilapia producer, pers. comm.), Egypt (M. ElNaggar, Mansoura University, pers. comm.), China (Anon., private tilapia producer, pers. comm.) and in Holland (C. Vancoillie, Zon-Aquafarming, pers. comm.) where the species in the latter seven cases were confirmed morphologically by the present authors to be $G$. cichlidarum Paperna, 1968. Gyrodactylus cichlidarum (Fig. 1a-g) was originally described from Sarotherodon galilaeus galilaeus (L.) (syn. Tilapia galilaea) from the Accra plains and Akuse lagoon, Lower Volta, Ghana, but has also been recorded from Tilapia zillii (Gervais) (syn. Chromis zillii), Hemichromis fasciatus Peters and H. bimaculatus Gill from various locations around the Volta Lake (Paperna 1968). Paperna (1979) cited additional hosts and locations for G. cichlidarum namely Sarotherodon melanotheron heudelotii (Duméril) and Tilapia guineensis (Günther) from coastal saline lagoons in Ghana; T. zillii, S. galilaeus galilaeus and Oreochromis aureus (Steindachner) from coastal Israel and Jordan systems; and Haplochromis flaviijosephi

Address for correspondence: A. García-Vásquez, Institute of Aquaculture, University of Stirling, Stirling FK9 4LA, Scotland, UK.

Phone: ++44 1786473 171; Fax: ++44 1786472 133; E-mail: ag22@stir.ac.uk 
(Lortert) and Tristamella simonis simonis (Günther) from systems in Jordan and Israel. The description of $G$. cichlidarum, however, along with many of the other African gyrodactylids that were made at the time are incomplete and require re-examination. An infection of gyrodactylids on yolk sac Oreochromis niloticus niloticus (L.) (syn. Tilapia niloticus) resulting in high mortalities in an isolated aquarium facility at the University of Stirling, therefore, provided an opportunity to collect material and to provide a new and detailed revised description of $G$. cichlidarum.

\section{MATERIALS AND METHODS}

Morphometrics. Yolk sac fry of $O$. n. niloticus bearing heavy infections of Gyrodactylus sp. were noted and collected from an isolated aquarium system at the University of Stirling, UK. Live specimens were prepared and fixed using ammonium picrate glycerine (APG) as detailed by Malmberg (1970). Further specimens were either air-dried directly onto slides or fixed in $80 \%$ ethanol and subsequently digested to release their hooks as detailed below.

The haptoral hard parts from 20 air-dried Gyrodactylus slide preparations were released using the proteolytic digestion method of Harris and Cable (2000), 5 specimens had their haptors excised and prepared for morphological studies while their bodies were fixed in $95 \%$ ethanol for molecular studies. The haptoral hard parts were studied at $\times 100$ magnification using oil immersion and a JVC KY-F30B 3CCD camera fitted to an Olympus $\mathrm{BH} 2$ compound microscope with an interfacing $\times 2.5$ top lens. Measurements were made on the attachment hooks using the Point-R macro (ver $1.0{ }^{\circ}$ University of Stirling, 2003) written through Zeiss KS300 iC/Windows release version 3.0. (1997) (Carl Zeiss Vision GmbH, München, Germany/Imaging Associates Ltd, Thame, Oxfordshire, UK) software. A total of 25 point-to-point measurements were made on each specimen $(\mathrm{n}=20)$ and voucher specimens were deposited in the helminth collections at The Natural History Musuem, London, the Institute of Parasitology, Academy of Sciences of the Czech Republic and in the Musée Royal de l'Afrique Centrale, Belgium. The holotype of G. cichlidarum (acc. no. 35584) from the Musée Royal de l'Afrique Centrale (MRAC), Tervuren, Belgium, and also the holotype of $G$. niloticus (acc. no. 084007) and the paratypes (acc. nos. 084008) from the USDA U.S. National Parasite Collection, Maryland, USA were also measured alongside APG and digested preparations of G. cichlidarum from the current study. Gyrodactylid material collected from $O$. n. niloticus from Gania de Pucté, Municipal de Chablé, Tabasco, Mexico provided courtesy of Dr. Victor Vidal-Martínez from the CINVESTAV, Mérida, Mexico were also measured. All the morphometric measurements which follow those described in Shinn et al. (2004), are given in micrometres as a mean followed by the range in parentheses and are detailed in Table 1.

Molecular analysis. The body of one gyrodactylid specimen collected from aquarium stock of $O$. $n$. niloticus in Stirling was prepared for sequencing according to Cunningham et al. (2001). The primer pairs ITS1A (5'-GTAACAAGGTTT CCGTAGGTG-3') and ITS2 (5'-TCCTCCGCTTAGTGATA3') (Matějusová et al. 2001) were used to amplify (PCR) a fragment spanning the 3 ' end of the $18 \mathrm{~S}$ subunit, the ITS1 and ITS 2 and the $5.8 \mathrm{~S}$ gene and the 5 ' end of the $28 \mathrm{~S}$ subunit. The reaction contained $1 \mu \mathrm{l}$ of DNA template, PCR buffer (Roche), $200 \mathrm{mM}$ dNTPs, $1 \mathrm{mM}$ of each primer and 1U Taq polymerase (Roche) in a total volume of $25 \mu 1$. The PCR reaction was performed in a GeneAmp PCR System 9700 (Applied Biosystems) using the following protocol: $4 \mathrm{~min}$ at $95^{\circ} \mathrm{C}$, followed by 35 cycles of $1 \mathrm{~min}$ at $95^{\circ} \mathrm{C}, 1 \mathrm{~min}$ at $50^{\circ} \mathrm{C}$ and 2 $\min$ at $72^{\circ} \mathrm{C}$. The PCR-products were purified using a QIAquick PCR Purification Kit (Qiagen) according to the manufacturer's recommendations. Both DNA strands were sequenced using BigDye chemistry (Applied Biosystems) and an ABI3100 automatic sequencer (Applied Biosystems). The PCR primers and the internal primers ITS1R (5'-ATTT GCGTTCGAGAGACCG-3') and ITS2F (5'-TGGTGGATCA CTCGGCTCA-3') (Ziętara and Lumme 2003) were used for sequencing.

\section{RESULTS}

\section{Gyrodactylus cichlidarum Paperna, 1968}

H o s t: Oreochromis niloticus niloticus (L.).

$\mathrm{S}$ i t e : Skin and fins.

L o c a 1 i t y : Cultured stock, University of Stirling, UK; Gania de Pucté, Municipal de Chablé, Tabasco, Mexico; holotype (acc. no. 35584) from Sarotherodon g. galilaeus from the Lower Volta, Ghana.

$\mathrm{M}$ a t e $\mathrm{r}$ i a 1 : Twenty proteolytic digested specimens and 10 APG mounted specimens (current study); holotype of $G$. cichlidarum (acc. no. 35584, MRAC). PCR amplified internal transcribed spacers (ITS) 1 and 2 and 5.8S gene sequences are deposited in GenBank under accession number DQ124228. Voucher specimens are deposited in the helminthological collection at the Natural History Museum, London (acc. nos. 2004.12.8.9-11; $\mathrm{n}=3$ ), Musée Royal de l'Afrique Centrale, Belgium (MRAC) (acc. nos. MRAC $37560-37562 ; n=3$ ) and the Institute of Parasitology, Academy of Sciences of the Czech Republic, České Budějovice (acc. no. $M-406 ; n=3$ ).

\section{Morphological description}

Figs. 1, 2a-c, e, 3; Table 1

Coverslip-flattened specimens 237.5-375 (313.5) long, 24.5-90 (72.7) wide at uterus. Anterior pharynx bulb 19.1-33.3 (25.9) long, 22.5-39.4 (30.5) wide, posterior pharynx bulb 11.7-28.4 (20.9) long, 22.6-44.4 (32.8) wide. Excretory bladders present. Gut not extending beyond level of ovary. Haptor round in shape and clearly delineated from body, length 67.5-87.5 (75.7), width 75-100 (89.2). Penis posterior to pharynx, 13.4 15.5 (14.3) (length) $\times 12.6-17.0$ (14.4) (width), armed with large apical spine 4.5-5.4 (5.0) long, one set of robust "terminal" spines, 1.6-2.5 (2.1), and two pairs of increasingly gracile "sub-terminal" and "medial" spines, 1.3-2.6 (1.9) and 0.7-2.6 (1.9) long respectively (Fig. $3 \mathrm{~h}-\mathrm{i})$. Total length of hamulus 46.6-59.9 (54.3); shaft 26.9-35.1 (32.7) long; point 23.3-27.9 (25.9) long; hamulus root 15.6-22.2 (19.7) long and represents 

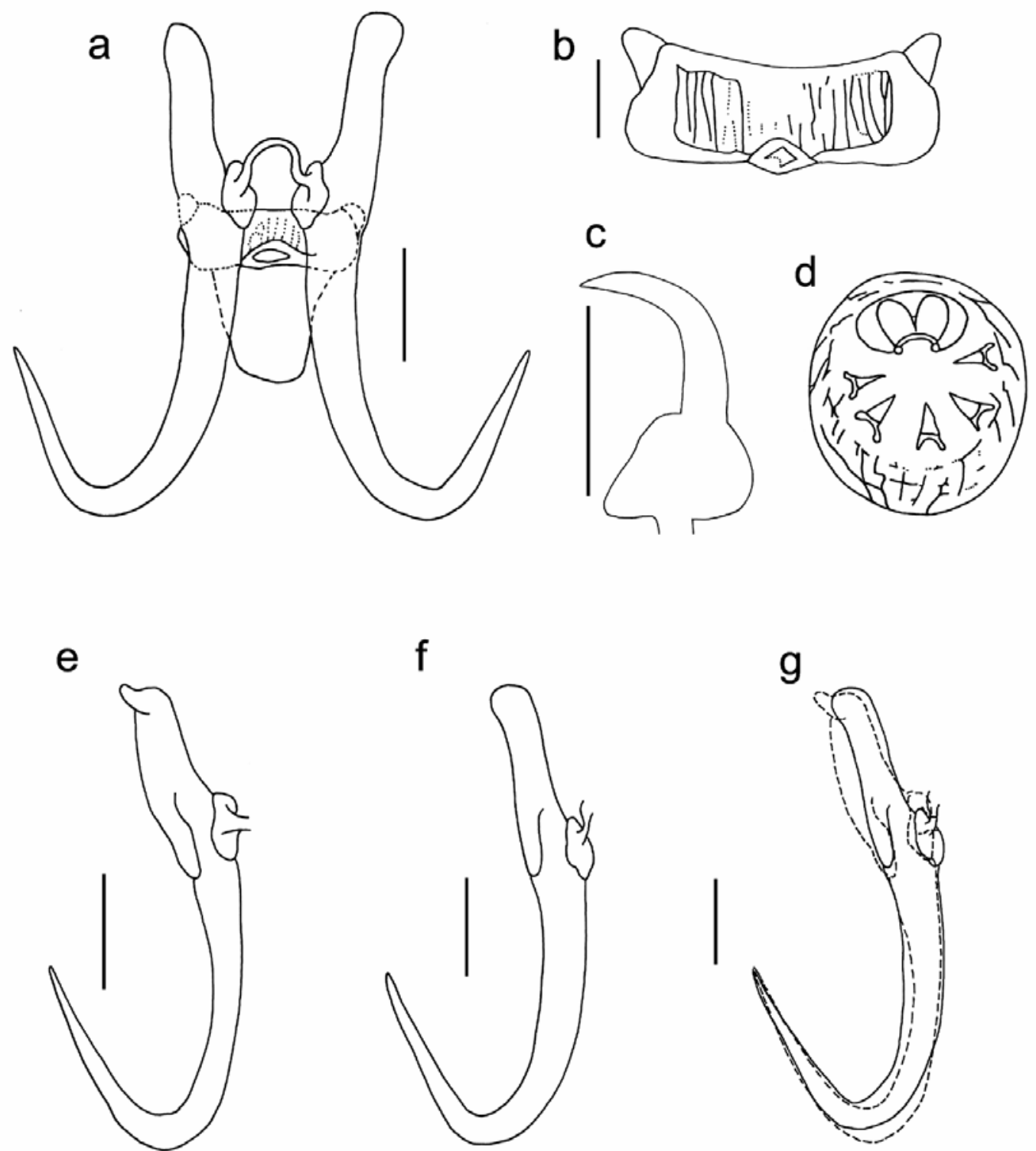

Fig. 1. Drawings of the opisthaptoral hard parts and penis of Gyrodactylus cichlidarum Paperna, 1968 from Sarotherodon galilaeus galilaeus. a - central hook complex, holotype (re-examination); b - ventral bar (redrawn from Paperna 1968); $\mathbf{c}-$ marginal hook sickle (re-examination); d - penis, Paperna's original drawing (no scale bar given in the original description); e hamulus (adapted from Paperna 1968); f - hamulus, holotype (re-examination); g - hamulus, overlay of Paperna's (1968) original drawing (dashed line) with that derived from the re-examination (solid line). Scale bars: $\mathrm{a}, \mathrm{b}, \mathrm{e}-\mathrm{g}=10 \mu \mathrm{m} ; \mathrm{c}=5 \mu \mathrm{m}$.

approximately one third of total length of hook; aperture angle of hamulus $41.2-56.5^{\circ}\left(45.0^{\circ}\right)$ with hamulus point terminating just below ventral bar attachment point giving the entire hook closed appearance; anterior edge of dorsal bar attachment point on hamulus is prominent creating characteristic notch between root and dorsal bar attachment point (Figs. 2c, 3c-d). Dorsal bar straight 1.2-1.8 (1.5) wide, 18.2-25.3 (21.5) long (Fig. 2a). Ventral bar 20.1-26.9 (22.8) wide, 19.4-25.7 (22.4) long, ventral bar processes 1.2-2.3 (1.8) long; ventral bar membrane 11.1-14.3 (12.9) almost half the length of hamulus shaft, approximately square in shape with medial, spatulate ridge. Lower edge of the median portion of ventral bar with characteristic crescent-shaped depression (Figs. 2b, 3b). Marginal hooks 24.3-30.5 (28.2) long; shaft length 17.8-22.8 (21.2) the end of which is spatulate; sickle proper 6.9-8.4 (7.4) long with deep sickle base (approximately 2-6), short triangular toe 1.1-1.8 (1.5) long, narrow bridge, short instep/arch $0.1-0.4(0.2)$, and rounded heel (Figs. 2e, 3f-g). The sickle blade describes shallow curve extending beyond limit of toe with point terminating at position that is parallel to sickle base. This gives the marginal sickle a very open aperture which measures 6.4-7.9 (6.9). Distally, sickle measures 4.1-6.3 (4.8) wide; proximally 3.9-5.2 (4.3) wide (Figs. 2e, 3f-g) with filament loop 10.9-15.1 (14.0) long approximately half the entire length of marginal hook. 


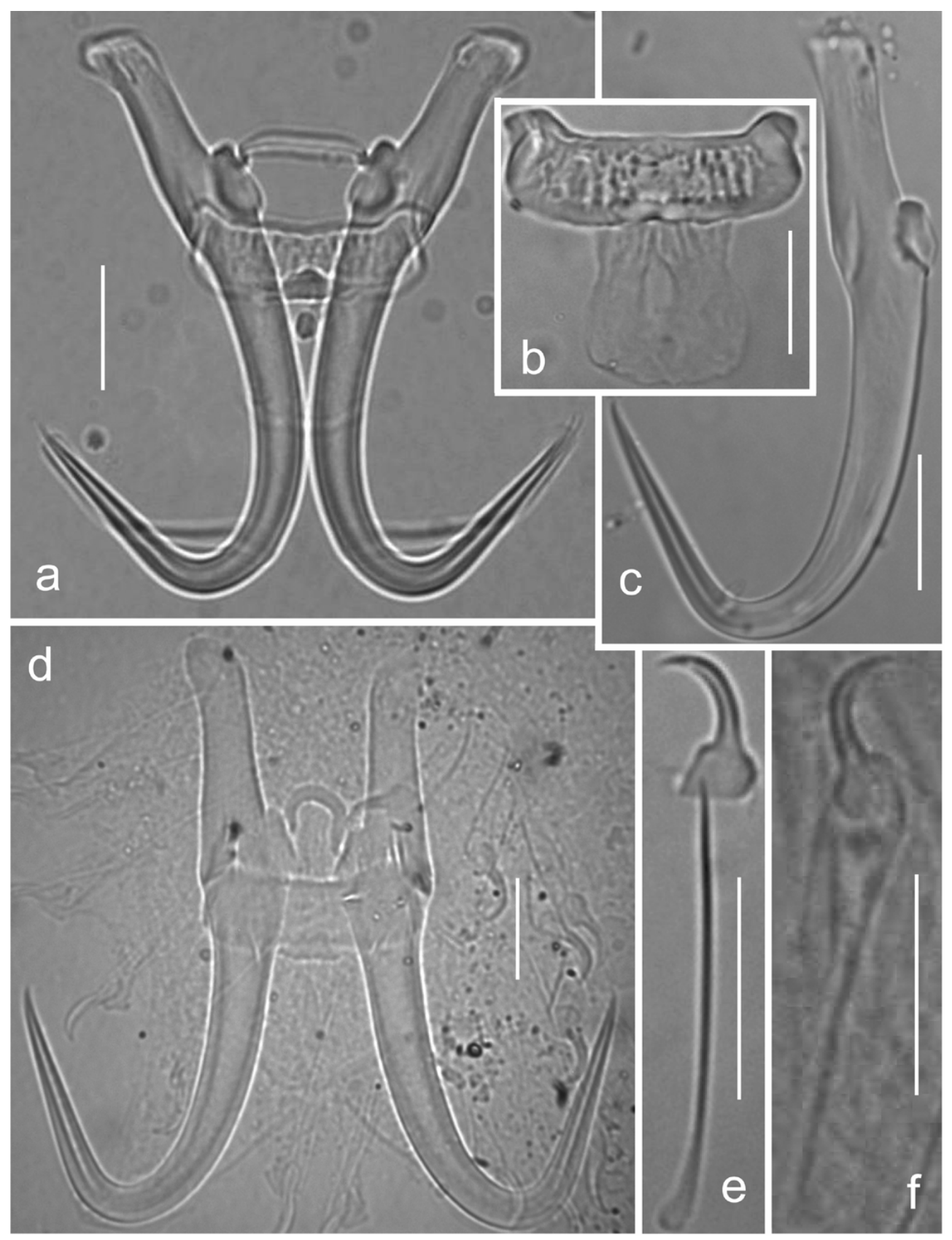

Fig. 2. Light micrographs of the opisthaptoral hard parts of Gyrodactylus cichlidarum Paperna, 1968 from Oreochromis niloticus niloticus (UK held stock) released by proteolytic digestion (a-c, e), compared with the holotype of Gyrodactylus niloticus Cone, Arthur et Bondad-Reantaso, 1995 (d, f). a - central hook complex; b - ventral bar; c - hamulus; d - central hook complex, $G$. niloticus; $\mathbf{e}$ - marginal hook; $\mathbf{f}$ - marginal hook, G. niloticus. Scale bars $=10 \mu \mathrm{m}$.

\section{Molecular characterisation}

The amplified nucleotide sequence of the rDNA cluster was $861 \mathrm{bp}$ and consisted of the $3^{\prime}$ end of the $18 \mathrm{~S}$ subunit, the ITS1 (343 bp) and ITS2 (303 bp) and the $5.8 \mathrm{~S}$ gene $(157 \mathrm{bp})$ and the 5 ' end of the $28 \mathrm{~S}$ subunit. A BLASTN (Altschul 1991) search of the sequence in GenBank (June 2005) revealed similar sequences belonging to the genus Gyrodactylus, but suggested that it was very divergent from the sequences of the other spe- cies deposited so far. The sequence shows large similarities with other species of Gyrodactylus within GenBank only in a conserved area of approximately $220 \mathrm{bp}$ spanning the last $20 \mathrm{bp}$ of ITS1, the complete 5.8S (157 bp), and the first 50 bp of ITS2. Submitting ITS1 to a BLAST search separately revealed no related sequences at all, and the conserved $5.8 \mathrm{~S}$ sequence is different by 8 nucleotides $(5.1 \%)$ to the nearest hit (Gyrodactylus sprostonae Ling, 1962, AY278044). 


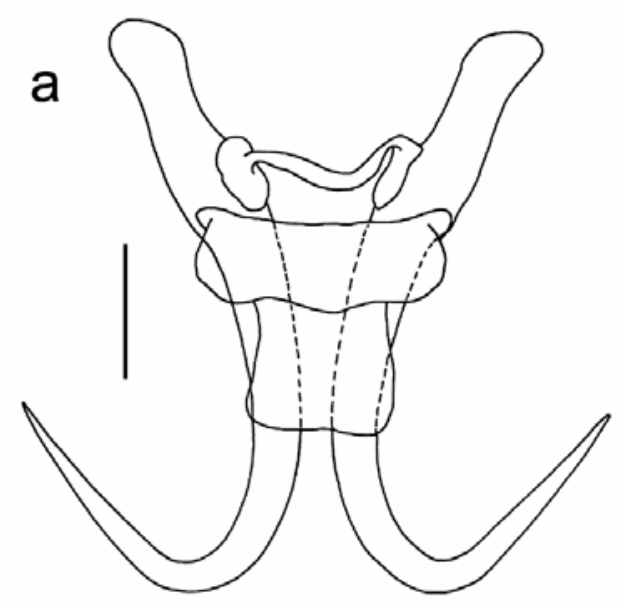

b
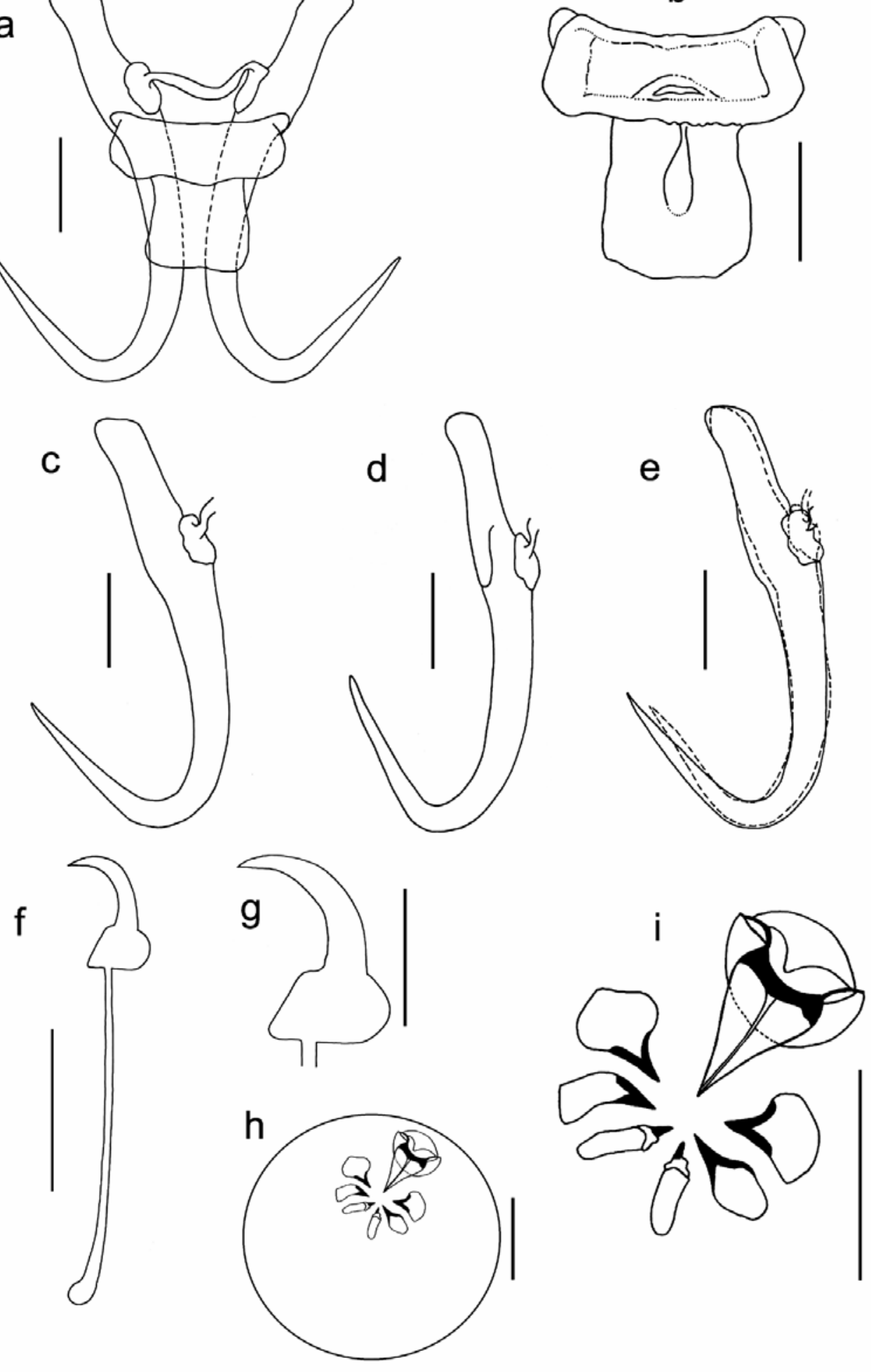

Fig. 3. Drawings of Gyrodactylus cichlidarum Paperna, 1968 from Oreochromis niloticus niloticus (UK held stock) (a-c, f-i), compared with Paperna's holotype (d). a - central hook complex; b - ventral bar; c - hamulus; d - hamulus, holotype (reexamination); e - overlay of "d" (dashed line) with "c" (solid line); $\mathbf{f}$ - marginal hook; $\mathbf{g}$ - marginal hook sickle; $\mathbf{h}$ - penis; $\mathbf{i}$ - arrangement of the penis spines. Scale bars: $\mathrm{a}-\mathrm{f}=10 \mu \mathrm{m} ; \mathrm{g}-\mathrm{i}=5 \mu \mathrm{m}$. 
Table 1. Morphological measurements of Gyrodactylus spp. collected from Oreochromis n. niloticus are compared with those originally reported by Paperna (1968) and with the new measurements made on the holotype of G. cichlidarum Paperna, 1968. Gyrodactylids collected from $O$. n. niloticus from the current study are compared with the measurements of $G$. niloticus Cone, Arthur et Bondad-Reantaso, 1995 and G. shariffi Cone, Arthur et Bondad-Reantaso, 1995 which are also known to parasitize $O$. $n$. niloticus. For each variable, the mean \pm standard deviation and the range in parentheses is given. ${ }^{a} \mathrm{n}=10,{ }^{\mathrm{b}} \mathrm{n}=5,{ }^{\mathrm{c}} \mathrm{n}=8,{ }^{\mathrm{d}} \mathrm{n}=7,{ }^{\mathrm{e}} \mathrm{n}=9,{ }^{\mathrm{f}} \mathrm{n}=2$.

\begin{tabular}{|c|c|c|c|c|c|c|c|}
\hline Measurement & $\begin{array}{l}\text { G. cichlidarum } \\
(\mathrm{n}=20) \\
\text { UK material }\end{array}$ & $\begin{array}{c}\text { G. cichlidarum } \\
(\mathrm{n}=6) \\
\text { Paperna } 1968\end{array}$ & $\begin{array}{c}\text { G. cichlidarum } \\
(\mathrm{n}=1) \\
\text { holotype } \\
\text { (re-examination) }\end{array}$ & $\begin{array}{c}\text { Gyrodactylus sp. } \\
(\mathrm{n}=2) \\
\text { UK material }\end{array}$ & $\begin{array}{c}\text { G. niloticus } \\
(\mathrm{n}=9) \\
\text { Cone et al. } 1995\end{array}$ & $\begin{array}{c}\text { G. niloticus } \\
(\mathrm{n}=3) \\
\text { (re-examination) }\end{array}$ & $\begin{array}{c}\text { G. shariffi } \\
(\mathrm{n}=10) \\
\text { Cone et al. } 1995\end{array}$ \\
\hline Total body length & $\begin{array}{l}313.5 \pm 49.9^{\mathrm{a}} \\
(237.5-375)\end{array}$ & $\begin{array}{c}- \\
(250-350)\end{array}$ & 387.5 & - & $\begin{array}{c}388.0 \pm 21.1 \\
(360-416)\end{array}$ & - & $\begin{array}{c}289.7 \pm 43.9 \\
(240-383)\end{array}$ \\
\hline Total body width & $\begin{array}{c}72.7 \pm 19.7^{\mathrm{a}} \\
(24.5-90)\end{array}$ & $\begin{array}{c}- \\
(60-100)\end{array}$ & 70 & - & $\begin{array}{c}87.1 \pm 24.5 \\
(64-128)\end{array}$ & - & $\begin{array}{c}76.6 \pm 13.5 \\
(48-96)\end{array}$ \\
\hline Haptor length $\times$ width & $\begin{array}{c}75.8 \pm 7.4^{\mathrm{a}} \\
(67.5-87.5)^{\times} \\
89.3 \pm 7.9 \\
(75-100)\end{array}$ & $\begin{array}{c}- \\
(50-60)\end{array}$ & $75 \times 85$ & - & (07 120) & - & $(70,0)$ \\
\hline $\begin{array}{l}\text { Pharynx anterior bulb length } \times \\
\text { width }\end{array}$ & $\begin{array}{c}25.9 \pm 6.2^{\mathrm{b}} \\
(19.1-33.3) \times \\
30.5 \pm 6.6 \\
(22.5-39.4)\end{array}$ & - & - & - & $\begin{array}{c}26.0 \pm 4.0^{\mathrm{c}} \\
(22-39) \times \\
27.9 \pm 4.6 \\
(24-35)\end{array}$ & - & $\begin{array}{c}26.2 \pm 6.0 \\
(20-39) \times \\
30.7 \pm 6.4 \\
(22-43)\end{array}$ \\
\hline $\begin{array}{l}\text { Pharynx posterior bulb length } \times \\
\text { width }\end{array}$ & $\begin{array}{c}20.9 \pm 8.1^{\mathrm{b}} \\
(11.7-28.4) \times \\
32.8 \pm 8.2 \\
(22.6-44.4)\end{array}$ & - & - & - & - & - & - \\
\hline Penis length $\times$ width & $\begin{array}{c}14.3 \pm 0.9^{\mathrm{b}} \\
(13.4-15.5) \times \\
14.4 \pm 1.8 \\
(12.6-17)\end{array}$ & - & - & - & $\begin{array}{c}11.4 \pm 1.9^{\mathrm{d}} \\
(10-14)\end{array}$ & - & $\begin{array}{l}10.1 \pm 1.1^{\mathrm{c}} \\
(9-12)\end{array}$ \\
\hline Hamulus total length & $\begin{array}{c}54.3 \pm 3.4 \\
(46.6-59.9)\end{array}$ & $\begin{array}{c}- \\
(10-80)\end{array}$ & 54.3 & $\begin{array}{c}55.7 \pm 0.2 \\
(55.5-55.8)\end{array}$ & $\begin{array}{c}61.3 \pm 2.3^{\mathrm{c}} \\
(59-66)\end{array}$ & $\begin{array}{c}58.3 \pm 0.9 \\
(57-59)\end{array}$ & $\begin{array}{c}45.3 \pm 1.6 \\
(44-48)\end{array}$ \\
\hline Hamulus aperture & $\begin{array}{c}22.5 \pm 1.5 \\
(17.1-24.2)\end{array}$ & - & 22.2 & $\begin{array}{c}15.5 \pm 0.2 \\
(15.3-15.6)\end{array}$ & $(0)=00)$ & $\begin{array}{c}21.4 \pm 0.4 \\
(20-22)\end{array}$ & - \\
\hline Hamulus proximal shaft & $\begin{array}{l}7.4 \pm 0.6 \\
(6.4-8.4)\end{array}$ & - & 7.7 & $\begin{array}{l}7.5 \pm 0.2 \\
(7.3-7.6)\end{array}$ & - & $\begin{array}{l}8.3 \pm 0.7 \\
(7-9)\end{array}$ & - \\
\hline Hamulus point length & $\begin{array}{c}25.9 \pm 1.3 \\
(23.3-27.9)\end{array}$ & $\stackrel{-}{(20-25)}$ & 24.3 & $\begin{array}{c}27.7 \pm 0.3 \\
(27.5-27.9)\end{array}$ & $\begin{array}{c}25.6 \pm 1.9 \\
(22-28)\end{array}$ & $\begin{array}{c}27.1 \pm 0.2 \\
(26-28)\end{array}$ & $\begin{array}{c}19.6 \pm 1.1^{\mathrm{e}} \\
(17-21)\end{array}$ \\
\hline Hamulus distal shaft width & $\begin{array}{l}4.5 \pm 0.6 \\
(3.3-5.7)\end{array}$ & - & 3.6 & $\begin{array}{c}5.4 \pm 0.5 \\
(5-5.6)\end{array}$ & - & $\begin{array}{c}4.1 \pm 0.3 \\
(3-5)\end{array}$ & - \\
\hline Hamulus shaft length & $\begin{array}{c}32.7 \pm 2.2 \\
(26.9-35.1)\end{array}$ & - & 29.2 & $\begin{array}{c}33.6 \pm 0.0 \\
(33.6-33.6)\end{array}$ & $\begin{array}{c}43.8 \pm 1.0 \\
(42-45)\end{array}$ & $\begin{array}{c}35.8 \pm 0.8 \\
(34-37)\end{array}$ & $\begin{array}{c}36.5 \pm 1.1 \\
(35-38)\end{array}$ \\
\hline Hamulus inner curve length & $\begin{array}{l}3.6 \pm 0.8 \\
(2.3-4.7)\end{array}$ & - & 3.1 & $\begin{array}{l}4.8 \pm 0.5 \\
(4.5-5.1)\end{array}$ & - & $\begin{array}{c}3.0 \pm 0.4 \\
(2-4)\end{array}$ & - \\
\hline Hamulus aperture angle $\left({ }^{\circ}\right)$ & $\begin{array}{l}45.0 \pm 3.6 \\
(38-50.1)\end{array}$ & - & 45.2 & $\begin{array}{c}30.0 \pm 1.8 \\
(28.7-31.2)\end{array}$ & - & $\begin{array}{c}39.6 \pm 0.3 \\
(39-40)\end{array}$ & - \\
\hline Hamulus point curve angle $\left({ }^{\circ}\right)$ & $\begin{array}{l}12.1 \pm 3.1 \\
(7.5-20.2)\end{array}$ & - & 11.4 & $\begin{array}{c}14.8 \pm 1.0 \\
(14.1-15.5)\end{array}$ & - & $\begin{array}{c}9.8 \pm 0.5 \\
(9-11)\end{array}$ & - \\
\hline Inner hamulus aperture angle $\left({ }^{\circ}\right)$ & $\begin{array}{c}52.3 \pm 4.1 \\
(41.2-56.5)\end{array}$ & - & 51.5 & $\begin{array}{c}36.7 \pm 2.7 \\
(34.8-38.5)\end{array}$ & - & $\begin{array}{c}44.7 \pm 0.6 \\
(44-46)\end{array}$ & - \\
\hline
\end{tabular}


Table 1. Continued.

\begin{tabular}{|c|c|c|c|c|c|c|c|}
\hline Measurement & $\begin{array}{l}\text { G. cichlidarum } \\
(\mathrm{n}=20) \\
\text { UK material }\end{array}$ & $\begin{array}{c}\text { G. cichlidarum } \\
(\mathrm{n}=6) \\
\text { Paperna } 1968\end{array}$ & $\begin{array}{c}\text { G. cichlidarum } \\
(\mathrm{n}=1) \\
\text { holotype } \\
\text { (re-examination) }\end{array}$ & $\begin{array}{c}\text { Gyrodactylus sp. } \\
(\mathrm{n}=2) \\
\text { UK material }\end{array}$ & $\begin{array}{c}\text { G. niloticus } \\
(\mathrm{n}=9) \\
\text { Cone et al. } 1995\end{array}$ & $\begin{array}{c}\text { G. niloticus } \\
(\mathrm{n}=3) \\
\text { (re-examination) }\end{array}$ & $\begin{array}{c}\text { G. shariffi } \\
(\mathrm{n}=10) \\
\text { Cone et al. } 1995\end{array}$ \\
\hline Hamulus root length & $\begin{array}{c}19.8 \pm 1.8 \\
(15.6-22.2)\end{array}$ & $\begin{array}{c}- \\
(15-20)\end{array}$ & 21.4 & $\begin{array}{c}20.6 \pm 0.1 \\
(20.5-20.7)\end{array}$ & $\begin{array}{c}20.6 \pm 2.6^{\mathrm{c}} \\
(17-25)\end{array}$ & $\begin{array}{c}22.4 \pm 0.7 \\
(21-23)\end{array}$ & $\begin{array}{c}10.8 \pm 2.3 \\
(8-14)\end{array}$ \\
\hline Ventral bar width & $\begin{array}{c}22.9 \pm 1.8 \\
(20.1-26.9)\end{array}$ & $\begin{array}{c}- \\
(5-8)\end{array}$ & 19.6 & $\begin{array}{c}22.3 \pm 0.2 \\
(22.1-22.4)\end{array}$ & $\begin{array}{c}21.1 \pm 0.9 \\
(20-22)\end{array}$ & $\begin{array}{c}22.0 \pm 2.0 \\
(20-24)\end{array}$ & $\begin{array}{c}20.2 \pm 1.0 \\
(18-22)\end{array}$ \\
\hline Ventral bar length & $\begin{array}{c}22.5 \pm 1.5 \\
(19.4-25.7)\end{array}$ & - & 19.2 & $\begin{array}{c}22.8 \pm 0.2 \\
(22.6-22.9)\end{array}$ & - & $\begin{array}{l}24.0 \pm 1.6 \\
(22-25)\end{array}$ & $\begin{array}{l}5.00 \pm 0.0 \\
(5)\end{array}$ \\
\hline $\begin{array}{l}\text { Ventral bar processes to mid- } \\
\text { length }\end{array}$ & $\begin{array}{l}2.1 \pm 0.6 \\
(1.3-3.3)\end{array}$ & & 1.2 & $\begin{array}{l}2.5 \pm 0.4 \\
(2.2-2.8)\end{array}$ & - & $\begin{array}{l}2.5 \pm 0.6 \\
(2-3)\end{array}$ & - \\
\hline Ventral bar median length & $\begin{array}{l}7.6 \pm 0.9 \\
(5.9-9.5)\end{array}$ & - & 5.4 & $\begin{array}{l}7.9 \pm 0.1 \\
(7.8-7.9)\end{array}$ & $\begin{array}{c}6.2 \pm 0.7 \\
(5-7)\end{array}$ & $\begin{array}{c}7.3 \pm 0.8 \\
(6-8)\end{array}$ & - \\
\hline Ventral bar process length & $\begin{array}{l}1.8 \pm 0.3 \\
(1.2-2.3)\end{array}$ & - & 1.5 & $\begin{array}{l}2.3 \pm 0.1 \\
(2.2-2.4)\end{array}$ & $\begin{array}{c}2 \pm 0.0 \\
(2)\end{array}$ & $\begin{array}{c}1.6 \pm 0.1 \\
(1-2)\end{array}$ & $\begin{array}{c}9.0 \pm 0.9^{\mathrm{e}} \\
(7-10)\end{array}$ \\
\hline Ventral bar membrane length & $\begin{array}{c}12.9 \pm 1.1 \\
(11.0-14.3)\end{array}$ & - & 12.9 & $\begin{array}{c}12.8 \pm 0.3 \\
(12.5-12.9)\end{array}$ & $\begin{array}{c}13.8 \pm 0.4^{\mathrm{c}} \\
(13-14)\end{array}$ & $\begin{array}{c}13.7 \pm 1.2 \\
(12-15)\end{array}$ & $\begin{array}{c}14.0 \pm 1.2 \\
(11-15)\end{array}$ \\
\hline Dorsal bar length & $\begin{array}{l}1.5 \pm 0.2^{\mathrm{b}} \\
(1.2-1.8)\end{array}$ & $\begin{array}{c}- \\
(1-2)\end{array}$ & 1.1 & - & - & - & $\begin{array}{c}17.0 \pm 2.0^{\mathrm{e}} \\
(14-20)\end{array}$ \\
\hline Dorsal bar width & $\begin{array}{l}21.5 \pm 3.0^{\mathrm{b}} \\
(18.2-25.3)\end{array}$ & $(16-22)$ & 19.0 & - & $\begin{array}{c}20.0 \pm 2.6^{\mathrm{c}} \\
(14-22)\end{array}$ & - & (2 +3 \\
\hline Marginal hook total length & $\begin{array}{c}28.2 \pm 1.4 \\
(24.3-30.3)\end{array}$ & $\begin{array}{c}- \\
(15-30)\end{array}$ & 27.9 & $\begin{array}{c}28.1 \pm 0.4 \\
(27.7-28.3)\end{array}$ & $\begin{array}{c}27.1 \pm 1.9^{\mathrm{c}} \\
(23-29)\end{array}$ & $\begin{array}{c}27.0 \pm 0.7 \\
(26-28)\end{array}$ & $\begin{array}{c}19.5 \pm 1.0 \\
(18-21)\end{array}$ \\
\hline Marginal hook shaft length & $\begin{array}{c}21.3 \pm 1.0 \\
(17.8-22.8)\end{array}$ & - & 21.6 & $\begin{array}{l}21.4 \pm 0.7 \\
(20.7-21.7)\end{array}$ & $\begin{array}{c}19.9 \pm 1.4^{\mathrm{c}} \\
(17-21)\end{array}$ & $\begin{array}{c}20.0 \pm 0.6 \\
(20-22)\end{array}$ & $\begin{array}{c}15.8 \pm 1.1 \\
(14-18)\end{array}$ \\
\hline Marginal hook sickle length & $\begin{array}{l}7.5 \pm 0.4 \\
(6.9-8.4)\end{array}$ & - & 6.5 & $\begin{array}{l}7.3 \pm 0.0 \\
(7.3)\end{array}$ & $\begin{array}{l}7.7 \pm 0.5 \\
(7-8)\end{array}$ & $\begin{array}{l}7.3 \pm 0.2 \\
(7-8)\end{array}$ & $\begin{array}{l}3.8 \pm 0.4 \\
(3-4)\end{array}$ \\
\hline $\begin{array}{l}\text { Marginal hook sickle proximal } \\
\text { width }\end{array}$ & $\begin{array}{l}4.4 \pm 0.3 \\
(3.9-5.2)\end{array}$ & - & 2.9 & $\begin{array}{l}4.3 \pm 0.2 \\
(4.1-4.4)\end{array}$ & $\begin{array}{c}3.8 \pm 0.5^{\mathrm{c}} \\
(3-4)\end{array}$ & $\begin{array}{c}4.3 \pm 0.2 \\
(4-5)\end{array}$ & $\begin{array}{c}3.2 \pm 0.4 \\
(3-4)\end{array}$ \\
\hline Marginal hook toe length & $\begin{array}{l}1.5 \pm 0.2 \\
(1.1-1.8)\end{array}$ & - & 1.1 & $\begin{array}{l}1.5 \pm 0.2 \\
(1.3-1.7)\end{array}$ & - & $\begin{array}{c}1.7 \pm 0.2 \\
(1-2)\end{array}$ & - \\
\hline Marginal hook sickle distal width & $\begin{array}{l}4.9 \pm 0.5 \\
(4.1-6.3)\end{array}$ & - & 3.9 & $\begin{array}{c}5.4 \pm 0.5 \\
(5-5.7)\end{array}$ & $\begin{array}{c}4.5 \pm 0.8^{\mathrm{c}} \\
\quad(4-6)\end{array}$ & $\begin{array}{c}4.6 \pm 0.2 \\
(4-5)\end{array}$ & $\begin{array}{c}3.2 \pm 0.4 \\
(3-4)\end{array}$ \\
\hline Marginal hook aperture & $\begin{array}{l}7.0 \pm 0.3 \\
(6.4-7.9)\end{array}$ & - & 6.8 & $\begin{array}{l}7.0 \pm 0.1 \\
(6.9-7.1)\end{array}$ & - & $\begin{array}{l}7.3 \pm 0.1 \\
(7-8)\end{array}$ & - \\
\hline Marginal hook instep/arch height & $\begin{array}{l}0.3 \pm 0.1 \\
(0.1-0.4)\end{array}$ & - & 0.2 & $\begin{array}{l}0.3 \pm 0.1 \\
(0.2-0.3)\end{array}$ & - & $\begin{array}{l}0.2 \pm 0.1 \\
(0.1-0.2)\end{array}$ & - \\
\hline $\begin{array}{l}\text { Marginal hook filament loop } \\
\text { length }\end{array}$ & $\begin{array}{c}14.0 \pm 1.7 \\
(10.9-15.1)\end{array}$ & - & - & - & $\begin{array}{c}10.0 \pm 1.4^{\mathrm{f}} \\
(9-11)\end{array}$ & - & $\begin{array}{c}7.6 \pm 1.3 \\
(6-10)\end{array}$ \\
\hline
\end{tabular}




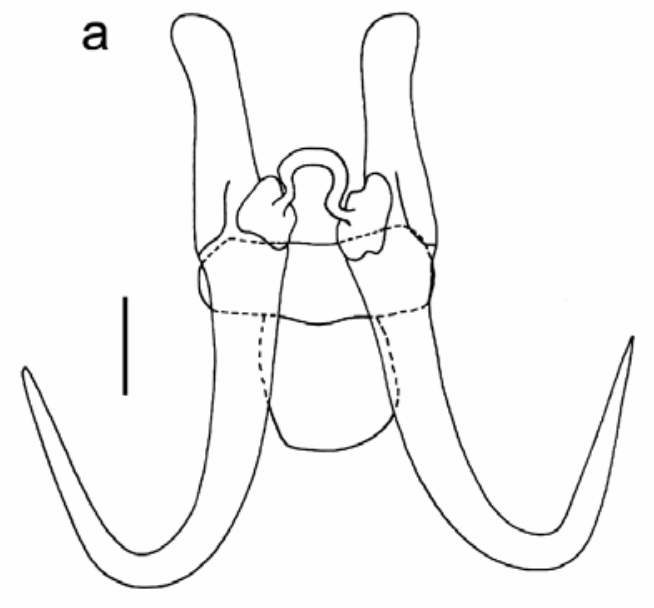

b
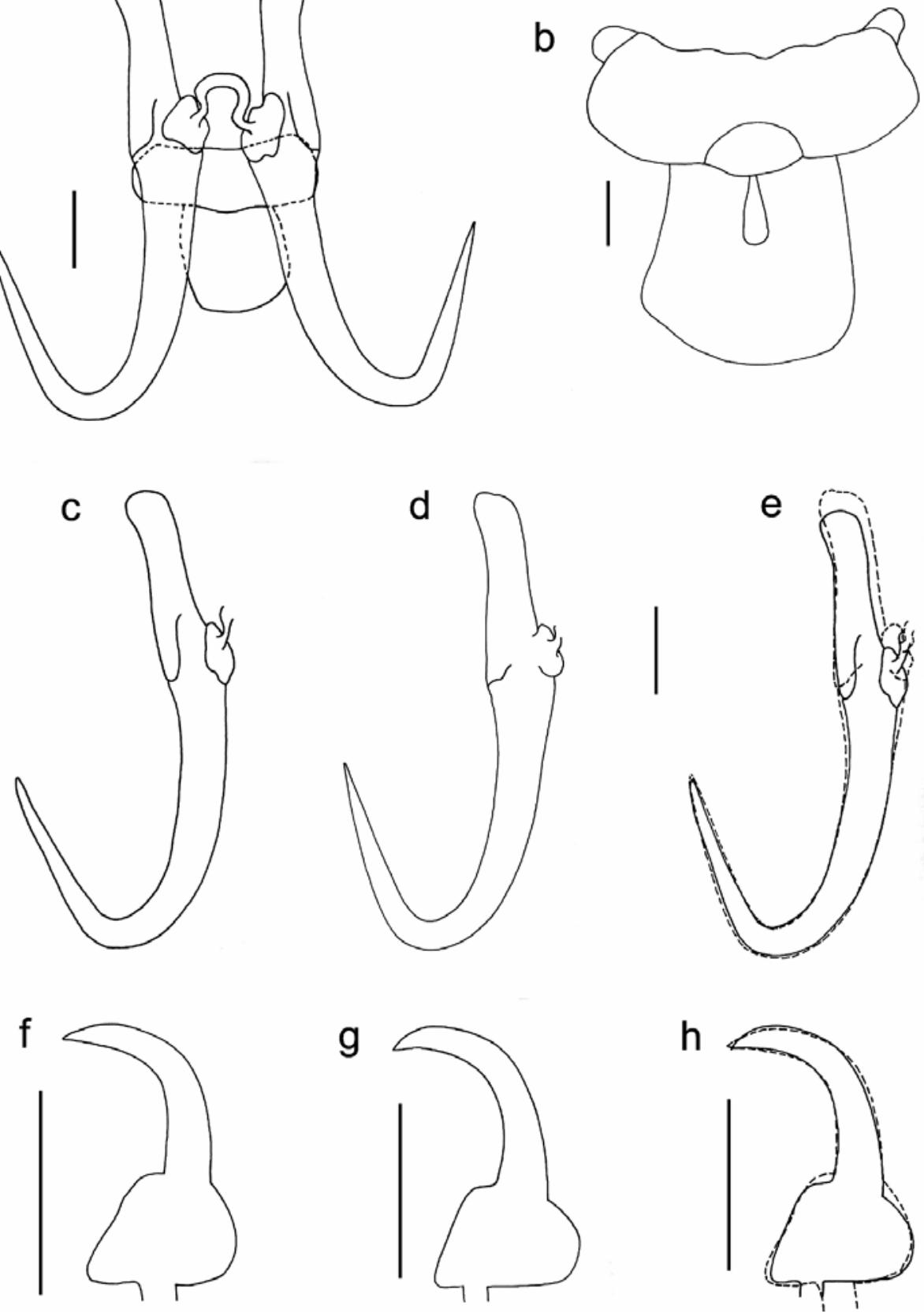

Fig. 4. Drawings of the opisthaptoral hard parts of the holotype and the paratype of Gyrodactylus niloticus Cone, Arthur et Bondad-Reantaso, 1995 (a, b, d, g), compared with G. cichlidarum (c, f). a - central hook complex (paratype), re-examination; $\mathbf{b}$ - re-drawing of ventral bar (paratype); c - hamulus of G. cichlidarum (holotype), re-examination; d - hamulus of G. niloticus (holotype), re-examination; e - overlay of "c" (solid line) with "d" (dashed line); f - marginal hook sickle of G. cichlidarum (holotype), re-examination; $\mathbf{g}$ - marginal hook sickle of G. niloticus (holotype), re-examination; $\mathbf{h}$ - overlay of " $\mathrm{f}$ " (dashed line) with "g" (solid line). Scale bars: $\mathrm{a}, \mathrm{c}-\mathrm{h}=10 \mu \mathrm{m} ; \mathrm{b}=5 \mu \mathrm{m}$.

\section{Gyrodactylus sp.}

H o s t: Oreochromis niloticus niloticus (L.). $\mathrm{S}$ i t e : Skin and fins.

L o c a lit y : Cultured stock, aquarium, Stirling, UK. M a t e r i a 1: Two proteolytic digested specimens.

\section{Description}

Fig. 5; Table 1

Coverslip-flattened specimens are similar in approximate dimensions to $G$. cichlidarum. The hamulus of Gyrodactylus sp. is similar to that of $G$. cichlidarum measuring 55.5-55.8 (55.6) in total length; shaft length 
33.5 (33.5); root 20.5-20.7 (20.6); (Fig. 5a, d, i), but differs in having a longer point 27.5-27.9 (27.7) and a smaller aperture angle $28.7-31.2^{\circ}\left(29.9^{\circ}\right)$ giving the hamulus a more closed appearance (Fig. 5k). Ventral bar 22.1-22.4 (22.3) wide; 22.6-22.9 (22.7) long; ventral bar processes 2.2-2.4 (2.3) long; shape of ventral bar membrane large and similar to that of G. cichlidarum, 12.5-12.9 (12.7) long (Fig. 5c, e). Marginal hooks 27.7-28.3 (28.0) long, shaft 20.7-21.7 (21.2) long; sickle proper 7.3 (7.3) long and 5-5.7 (5.3) distally and 4.1-4.4 (4.3) proximally in width (Fig. 5b, f). Marginal hook differs from that of $G$. cichlidarum by having shorter sickle shaft but longer point, which drops at its terminus giving the blade more rounded, apparently closed curve (Fig. 5h). Unfortunately the bodies of both specimens were unavailable for molecular analysis.

\section{Comments}

The holotype of G. cichlidarum from S. g. galilaeus deposited in the Musée Royal de l'Afrique Central, Belgium, represents the only preserved reference material for this species from Paperna's original work in Africa. The holotype had been mounted in Canada balsam and as a thick preparation, many of the smaller structures were difficult to observe and only the principle diagnostic features were commented upon and measured. The key diagnostic feature, however, the morphology of the marginal hooks was not described and the current study provides additional information in support of Paperna's original work. As it appears that G. cichlidarum is frequently encountered in facilities culturing tilapia, it seemed appropriate to re-examine material and to provide a revised description. As can be seen from Table 1, there are numerous inconsistencies between Paperna's original measurements on the holotype and those determined in the current study. Specifically, the size range of the hamulus total length reported by Paperna appears to be overly large at 10 to $80 \mu \mathrm{m}$ while those of the current study suggests that a total length of 46 to $60 \mu \mathrm{m}$ (54.3 for the holotype) is correct. Other disagreements in the measurements on the holotype include the ventral bar width. Paperna (1968) gives this measurement as 5 $8 \mu \mathrm{m}$ while the current study gives this as $19.6 \mu \mathrm{m}$. Much of the disparity in the measurements of this parameter, however, is linked to the different ways in which this structure has been measured. Malmberg (1970) gives the total width of the ventral bar as the distance between the ventral bar processes and the extremity of the membrane while Shinn et al. (2001) calls this measurement the total length. According to Malmberg (1970), the total width of the ventral bar can be broken down into the "width" of the median portion (not including the processes) and the "length" of the membrane. The finding from the current study would suggest that the "length" of the median portion (as cited in Shinn et al. 2001) of the ventral bar at $7.6 \pm 0.9 \mu \mathrm{m}$ is equivalent to the measurement intended by Paperna. The last discrepancy in the measurement of the holotype is in the measurement of the marginal hook total length. The range of measurements given by Paperna (1968) appear to be too large at $15-30 \mu \mathrm{m}$ cf. $27.9 \mu \mathrm{m}$ (holotype current study) which suggests that flexed hooks or the hooks of embryos were also included in the measurements. Alternatively, this could suggest that the original sample was a mix of species. The number and arrangement of the penial spines also differs between the two studies. Paperna (1968) describes the penis (syn. cirrus pouch) as having a large apical spine and five spines arranged in a single arch. The drawing given in the paper, however, suggests that the two terminal spines are smaller than the three medial spines (Fig. 1d). Although no penis was evident on the holotype; the findings from the current study suggest the penis of $G$. cichlidarum consists of one large apical hook and three sets of spines which although approximately equal in length, become increasingly gracile towards the centre line of the penis (Fig. $3 \mathrm{~h}-\mathrm{i})$. These observations $(\mathrm{n}=5)$ are supported by the material obtained from Mexico (n =2) (data not presented in Table 1). In addition to the measurements already commented upon, there are also some subtle differences in the shape of the hooks between those presented in Paperna (1968) and those of the current study (see Figs. 1c, e-g, 3c-g).

The ITS sequence of $G$. cichlidarum is the first sequence from a Gyrodactylus species originating from the African continent. The sequence is shorter and very divergent from other species sequenced so far, and only the 5.8S sequence places it unambiguously in Gyrodactylus. The $5.8 \mathrm{~S}$ sequence is very conserved in the genus and has been suggested to coincide with the subgenera that were proposed by Malmberg (1970) based on the characteristics of the excretory system (Ziętara et al. 2002, Ziętara and Lumme 2004) but Matějusová et al. (2003) and Huyse et al. (2003) gave less support to this view. The $5.8 \mathrm{~S}$ gene of G. cichlidarum, however, differs by more than $5.1 \%(8 / 157 \mathrm{bp})$ from the nearest hit in GenBank which could indicate that it belongs to a new subgenus. Considering the known (Harris et al. 2004) and suggested (Bakke et al. 2002) diversity within the genus Gyrodactylus and the fact that no other species from Africa have been sequenced, this would not be surprising. The study and classification of the parasites into subgenera based on the excretory system is performed on live specimens but despite numerous specimens of G. cichlidarum being available at the time of the infection, almost all the material was fixed in alcohol and the mapping of the excretory system was not done.

The first $50 \mathrm{bp}$ of ITS2 are identical to other Gyrodactylus species, but this is a conserved area also shared by close relatives e.g. Gyrdicotylus gallieni Vercammen-Grandjean, 1960 (AJ001843) and species in the genus Acanthoplacatus Ernst, Jones et Whittington, 2001, both in the family Gyrodactylidae. However, when the ITS1 sequence was submitted to a BLAST 

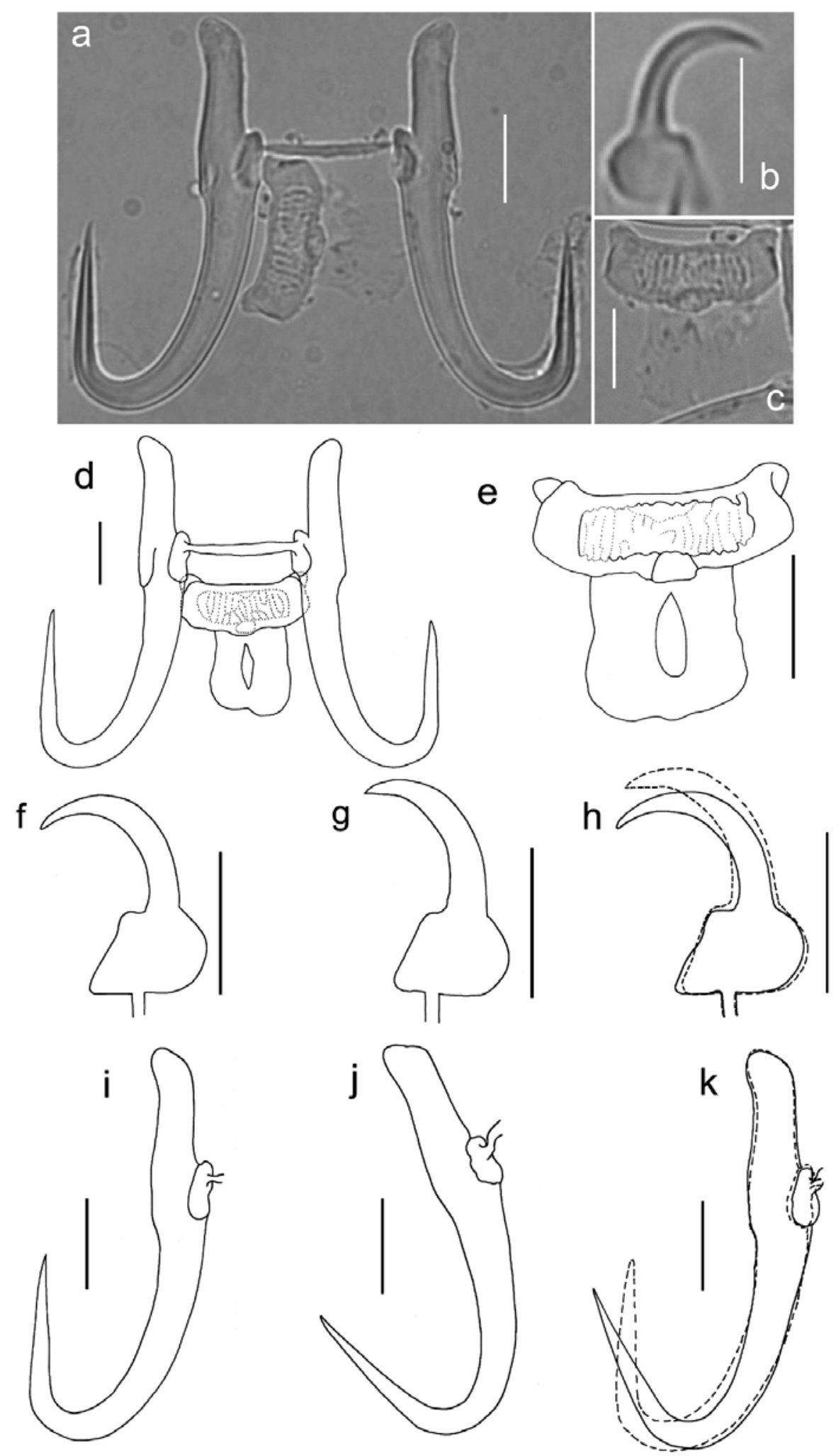

Fig. 5. Light micrographs and drawings of the opisthaptoral hard parts released by proteolytic digestion from specimens of Gyrodactylus sp. from Oreochromis niloticus niloticus (UK held stock) (a-f, i), compared with G. cichlidarum (same host and stock) (g, j). a, d - central hook complex; b - marginal hook sickle; c, e - ventral bar; f - marginal hook sickle; $\mathbf{g}$ - marginal hook sickle of G. cichlidarum; $\mathbf{h}$ - overlay of "g" (dashed line) with "b" (solid line); $\mathbf{i}$ - hamulus of Gyrodactylus sp.; $\mathbf{j}$ - hamulus of G. cichlidarum; $\mathbf{k}$ - overlay of "i" (dashed line) with "j" (solid line). Scale bars: $\mathrm{a}-\mathrm{e}, \mathrm{i}-\mathrm{k}=10 \mu \mathrm{m} ; \mathrm{f}-\mathrm{h}=5 \mu \mathrm{m}$. 
search in GenBank, no identical hits were obtained; a similar situation as has also been found for the ITS1 sequence of Gyrodactylus markakulensis Gvosdev, 1950 (Ziętara and Lumme 2004).

The fragment containing ITS1, 5.8S and ITS2 varies in length from 860 (G. carassii Malmberg, 1957, acc. no. AY278033) to 1,273 bp (G. teuchis Lautraite, Blanc, Thiery, Daniel et Vigneulle, 1999, acc. no. AJ249350) in the species of Gyrodactylus sequenced so far (Ziętara and Lumme 2004), but G. cichlidarum with only 803 bp is considerably shorter than this. The length of ITS1 has been shown to be phylogenetically informative (Cable et al. 1999, Matějusová et al. 2001, Ziętara et al. 2002, Ziętara and Lumme 2004) and Gyrodactylus species can be grouped according to the length of their ITS1. The 343 bp ITS1 of G. cichlidarum is of the "short type" (Ziętara and Lumme 2004), and this would group it with species that have been assigned to Gyrodactylus subgenera Gyrodactylus Malmberg, 1964, Metanephrotus Malmberg, 1964, Mesonephrotus Malmberg, 1964 and to the two species groups of Paranephrotus Malmberg, 1964, as well as the 'outgroup', Gyrodactyloides bychowskii Albova, 1948 (Ziętara and Lumme 2004). A detailed phylogenetic study would be necessary to infer the relationship of $G$. cichlidarum to other species in the genus, but this was, however, outside the scope of this study.

The haptoral hooks of two specimens, however, were found to differ from those of G. cichlidarum and these specimens will be referred to as Gyrodactylus sp. The latter species was observed to have a shorter hamulus aperture distance at $15.5 \mu \mathrm{m}$ cf. $22.5 \mu \mathrm{m}$ (G. cichlidarum), a smaller hamulus aperture angle $30.0^{\circ}$ (Gyrodactylus sp.) cf. $45.0^{\circ}$ (G. cichlidarum) and a smaller inner hamulus aperture angle $36.7^{\circ}$ (Gyrodactylus sp.) cf. $52.3^{\circ}$ (G. cichlidarum) (Fig. 5k). The only other observed differences were seen in the morphology of the marginal hook, notably in the shaft and point regions (Fig. 5h). As only two such specimens were encountered from over 100 that were examined in detail, Gyrodactylus sp. might represent, either another species or, more likely, a variant of $G$. cichlidarum. A molecular analysis of these two specimens would have shown whether these were morphological variants of G. cichlidarum or actually representing another species, but unfortunately the two specimens were mounted as whole mounts and were not available for molecular analysis.

The overlay of the hamuli and the marginal hooks confirm the identity of the gyrodactylids collected in the current study as G. cichlidarum. As the original marginal hook drawings from Paperna (1968) were not studied in detail and were not presented consistently they are, therefore, not represented here.

From the literature, two other species of Gyrodactylus are also known to parasitize O. n. niloticus (syn. Tilapia nilotica), viz. Gyrodactylus niloticus Cone, Arthur et Bondad-Reantaso, 1995 and G. shariffi Cone,
Arthur et Bondad-Reantaso, 1995, both of which were described from fish farms in the Philippines (Cone et al. 1995). To what extent these species also constitute a problem in tilapia hatcheries within the Philippines is unknown. Of these two species, however, the hooks of G. niloticus most closely resembled those of G. cichlidarum and, therefore, were studied in greater detail.

However, when comparing the drawings presented in the literature for $G$. niloticus with those made for $G$. cichlidarum, there appear to be several differences between both species. For example, the membrane of the ventral bar of $G$. niloticus appears to be square in its proportions, apparently lacking G. cichlidarum's characteristic depression in the median portion and the medial ridge along the membrane. However, a re-examination of the holotype (acc. no. 084007) and several paratypes (acc. no. 084008) shows that the morphology of the ventral bar of $G$. niloticus is identical to that of $G$. cichlidarum (cf. Fig. 3b with Fig. 4b). Similarly, a comparison of the marginal hooks initially suggested differences but, a re-examination of the holotypes of both species and an overlay of the hooks (Fig. 4h) confirmed that the morphology of the sickles of both species is identical. This latter observation is also supported by the point-to-point measurements (Table 1) and the identical morphology of the hamuli (Figs. 3e, 4e).

In conclusion, this study has provided additional information to support the original description of G. cichlidarum through the study of the holotype and new material, and a revised description is based on the examination of this new material. An error in the hamulus length reported by Paperna (1968) led to the creation of $G$. niloticus, however, the re-examination of material presents strong evidence to suggest the synonymy of $G$. cichlidarum with $G$. niloticus. Interestingly, it would appear that $G$. cichlidarum has achieved a wide distribution through the movement and culture of stocks of certain tilapine species in the same way as seen for $G$. salaris on rainbow trout, Oncorhynchus mykiss (Walbaum) (Malmberg and Malmberg 1993, Koski and Malmberg 1995). Cone et al. (1995) speculated that this parasite was delivered to the Philippines via the importation of tilapia and that $G$. shariffi was picked up from endemic mugilids. A continuation of this study attempts to make a worldwide morphological and molecular study of Gyrodactylus from Nile tilapia stocks and closely related species which, we hope, will provide further insight in to the geographical distribution of this genus of parasites and the morphological and genetic variation that may exist between stocks.

Acknowledgements. We would like to thank Dr. Rudy Jocqué from the Musée Royal de l'Afrique Centrale (MRAC) for the loan of the G. cichlidarum holotype from the Lower Volta, Ghana and Patricia Pilitt from the USDA U.S. National Parasite Collection for the loan of the G. niloticus holotype and paratypes from the Philippines. Particular thanks are also due to Dr. Victor Vidal-Martínez from CINVESTAV, Mérida, Mexico, Professor Mohammed El-Naggar from Mansoura 
University, Egypt, Caroline Vancoillie from Zon-Aquafarming, Holland, Warren Turner from Thailand and Nir Froyman from Israel for the collection of G. cichlidarum in support of this study. We are also grateful to the staff of the Tropical Aquarium at the University of Stirling for providing infected fish material for study. $\mathrm{HH}$ was supported by the Norwegian Research Council's Wild Salmon Programme (project no. 145861/720) and the National Centre for Biosystematics (project no. 146515/420) co-funded by the NRC and the NHM University of Oslo, Norway.

\section{REFERENCES}

ALTSCHUL S.F. 1991: Amino acid substitution matrices from an information theoretic perspective. J. Mol. Biol. 219: 555-565.

BAKKE T.A., HARRIS P.D., CABLE J. 2002: Host specificity dynamics observations on gyrodactylid monogeneans. Int. J. Parasitol. 32: 281-308.

CABle J., HARRIS P.D., Tinsley R.C., LAZARus C.M. 1999: Phylogenetic analysis of Gyrodactylus spp. (Platyhelminthes: Monogenea) using ribosomal DNA sequences. Can. J. Zool. 77: 1439-1449.

Cone D.K., Arthur R., BondAD-Reantaso M.G. 1995: Description of two new species of Gyrodactylus von Nordmann, 1832 (Monogenea) from cultured Nile tilapia, Tilapia nilotica (Cichlidae), in the Philippines. J. Helminthol. Soc. Wash. 62: 6-9.

Cunningham C.O., Mo T.A., Collins C.M., Buchmann K., THIERY R., BLANC G., LAUTRAITE A. 2001: Redescription of Gyrodactylus teuchis Lautraite, Blanc, Thiery, Daniel \& Vigneulle, 1999 (Monogenea: Gyrodactylidae): a species identified by ribosomal RNA sequence. Syst. Parasitol. 48: 141150 .

CUTLAND L. 2003: Tilapia: The world's most popular fish. IntraFish publication, http://www.intrafish.com

FAO Fishstat plus 2004: Universal software for fishery statistical time series $2004 \mathrm{FAO}$, http://www.fao.org/fi/statist/FISOFT/ FISHPLUS.asp

FITZSIMMONS K. 2000: Tilapia aquaculture in Mexico. In: B.A. Costa-Pierce and J.E. Rakocy (Eds.), Tilapia Aquaculture in the Americas, Vol. 2. The World Aquaculture Society, BatonRouge, Louisiana, United States, pp. 171-183.

FRYER G., ILES TD. 1972: The Cichlid Fishes of the Great Lakes of Africa: Their Biology and Evolution. T.F.H. Publ., Neptune City, New Jersey, 656 pp.

HARRIS P.D., CABLE J. 2000: Gyrodactylus poeciliae n. sp. and G. milleri n. sp. (Monogenea: Gyrodactylidae) from Poecilia caucana (Steindachner) in Venezuela. Syst. Parasitol. 47: 7985.

HARRIS P.D., SHINN A.P., CABLE J., BAKKE T.A. 2004: Nominal species of the genus Gyrodactylus von Nordmann 1832 (Monogenea: Gyrodactylidae), with a list of principal host species. Syst. Parasitol. 51: 1-27.

HuYse T., AudenaerT V., VolCKAERT F.A.M. 2003: Speciation and host-parasite relationships in the parasite genus Gyrodactylus (Monogenea, Platyhelminthes) infecting gobies of the genus Pomatoschistus (Gobiidae, Teleostei). Int. J. Parasitol. 33: 1679-1689.

KosKi P., MALMBerg G. 1995: Occurrence of Gyrodactylus (Monogenea) on salmon and rainbow trout in fish farms in Northern Finland. Bull. Scand. Soc. Parasitol. 5: 76-88.
MALMBERG G. 1970: The excretory systems and the marginal hooks as a basis for the systematics of Gyrodactylus (Trematoda, Monogenea). Ark. Zool. 23: 1-235.

MALMBERG G., MALMBERG M. 1993: Species of Gyrodactylus (Platyhelminthes, Monogenea) on salmonids in Sweden. Fish. Res. 17: 59-68.

MatěJusová I., Gelnar M., MCBeath A.J.A., Collins C.M., CUNNINGHAM C.O. 2001: Molecular markers for gyrodactylids (Gyrodactylidae: Monogenea) from five fish families (Teleostei). Int. J. Parasitol. 31: 738-745.

MatěJusová I., Gelnar M., Verneau O., CunNingham C.O., LITTLEWOOD D.T.J. 2003: Molecular phylogenetic analysis of the genus Gyrodactylus (Platyhelminthes: Monogenea) inferred from rDNA ITS region: subgenera versus species groups. Parasitology 127: 603-611.

PAPERNA I. 1968: Monogenetic trematodes collected from freshwater fish in Ghana. Second report. Bamidgeh 20: 88-90.

PAPERNA I. 1979: Monogenea of Inland Water Fishes in Africa. Musée Royal de l'Afrique Centrale, Tervuren, Serie 8, 226, $127 \mathrm{pp}$.

Roberts R., SOMMERVILle C. 1980: Diseases of tilapia. In: R.S.V. Pullin and R.H. Lowe-McConnell (Eds.), The Biology and Culture of Tilapias. ICLARM Conference Proceedings, Manila, pp. 247-263.

SHINN A.P., GIBSON D.I., SommERVILle C. 2001: Morphometric discrimination of Gyrodactylus salaris Malmberg (Monogenea) from species of Gyrodactylus parasitising British salmonids using novel parameters. J. Fish Dis. 24: 83-97.

SHINN A.P., HANSEN H., OLSTAD K., BACHMANN L., BAKKE T.A. 2004: The use of morphometric characters to discriminate species of laboratory-reared and wild populations of Gyrodactylus salaris and G. thymalli (Monogenea). Folia Parasitol. 51: 239-252.

ZIĘTARA M.S., HuYse T., LUMME J., VOLCKAERT F.A.M. 2002: Deep divergence among subgenera of Gyrodactylus inferred from rDNA ITS region. Parasitology 124: 39-52.

ZIĘTARA M.S., LUMME J. 2003: The crossroads of molecular, typological and biological species concepts: two new species of Gyrodactylus Nordmann, 1832 (Monogenea: Gyrodactylidae). Syst. Parasitol. 55: 39-52.

ZIĘTARA M.S., LUMME J. 2004: Comparison of molecular phylogeny and morphological systematics in fish parasite genus Gyrodactylus Nordmann, 1832 (Monogenea, Gyrodactylidae). Zool. Pol. 49: 5-28.

Accepted 8 January 2007 